\title{
Representation Challenges: The Reasons of the Research
}

\author{
Andrea Giordano \\ Michele Russo \\ Roberta Spallone
}

Augmented Reality (AR) and Artificial Intelligence (Al) are technological domains that closely interact with space at architectural and urban scale in the broader ambits of cultural heritage and innovative design. The growing interest is perceivable in many fields of knowledge, supported by the rapid development and advancement of theory and application, software and devices, fueling a pervasive phenomenon within our daily lives. These technologies demonstrate to be best exploited when their application and other information and communication technology (ICT) advancements achieve a continuum. In particular, AR defines an alternative path to observe, analyze and communicate space and artifacts. Besides, Al opens future scenarios in data processing, redefining the relationship between man and computer.

In the last few years, the AR/AI expansion and relationship have raised deep trans-disciplinary speculation. The research experiences have shown many cross-relations in Architecture and Design domains. Representation studies could arise an international debate as a convergence place of multidisciplinary theoretical and applicative contributions related to architecture, city, environment, tangible and intangible Cultural Heritage.

A research synergy between different Universities (Politecnico di Torino, University of Padua, Sapienza University of Rome) was the first occasion to share collected experiences. The discussion between scholars led to the importance of fixing a first state of the art about AR/AI topics in the national Representation area, analyzing the role of AR/AI in the chain of knowledge. Besides, critical interest has been focused on exploring the disciplinary boundaries, identifying affine disciplines involved in similar research, and activating 
effective relationships. Working beyond the usual research edges meant evaluating whether our discipline could expand its field of action, establishing as challenge new directions of research, possible partners, priorities, and objectives.

Our initiative started in the fall of 2019, with a series of international events related to research works based on possible intersections between $A R$ and $A l$ and the world of Representation. The importance of opening a discussion with our colleagues at national level was confirmed during these events, which were supported by Francesca Fatta [I], who advocated a meeting initiative. So, we created a first working group, formalized in the Scientific Committee, which includes the promoters: Francesca Fatta, Salvatore Barba, Marco Giorgio Bevilacqua, Stefano Brusaporci, Alessandro Luigini, Cettina Santagati and Alberto Sdegno. This group discusses the possibility to organize a Symposium intended as a national meeting framed in the Representation field, which took place online on 13 and 14 October 2020, managed by the Zoom platform of the University of Padua (fig. I). The call for this virtual event met the interest of 175 authors, mostly coming from national Universities, proposing 83 research experiences. The proposed topics were divided into three main areas of interest: Augmented Reality, Artificial Intelligence, Virtual Reality \& Digital Modeling. The related abstracts were reviewed and divided into 44 oral and 39 video presentations. This division wanted to preserve the duration of the event (two days) giving to everyone the opportunity to present their research. The video presentations were uploaded before the beginning of the conference on a dedicated YouTube channel and shared with all Symposium participants. In addition, the final recordings of the event were uploaded on the same channel, ensuring that all contributions could be reviewed. The Symposium counted 265 scholars during the two days (fig. 2).The meeting days were structured with plenary communications, proposing eight thematic sessions. After a brief introduction by the UID President (Francesca Fatta) and by the three organizers, each day featured four sessions, opened by a keynote speaker. The seven invited speakers belonging to different disciplinary fields showed the potential of $A R$ and $A l$ in their specific discipline, highlighting, on the one hand, the possible connections with the field of Representation while confirming the value of the transdisciplinary nature of our studies. The invited speakers were Mario Rasetti (ISI Foundation), Claudio Casetti (Politecnico di Torino), Michele Bonino (Politecnico di Torino), Simone Milani (University of Padua), Fabrizio Lamberti (Politecnico di Torino), Eleonora Grilli (Fondazione Bruno Kessler - FBK), Alberto Tono (Computational Design Studio - Studio HOK).

Fig. I. Snapshot of the Zoom platform during the conference.

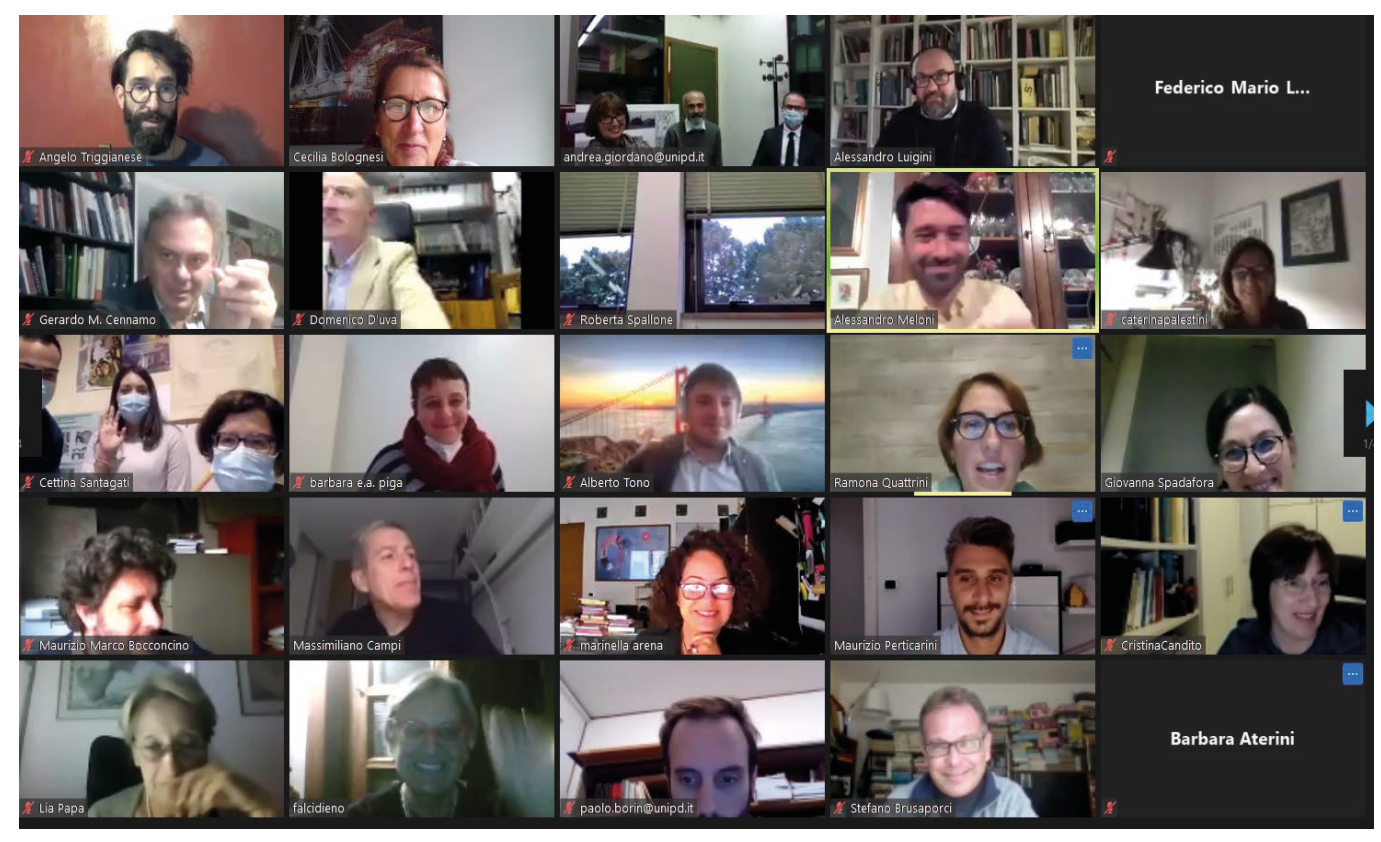




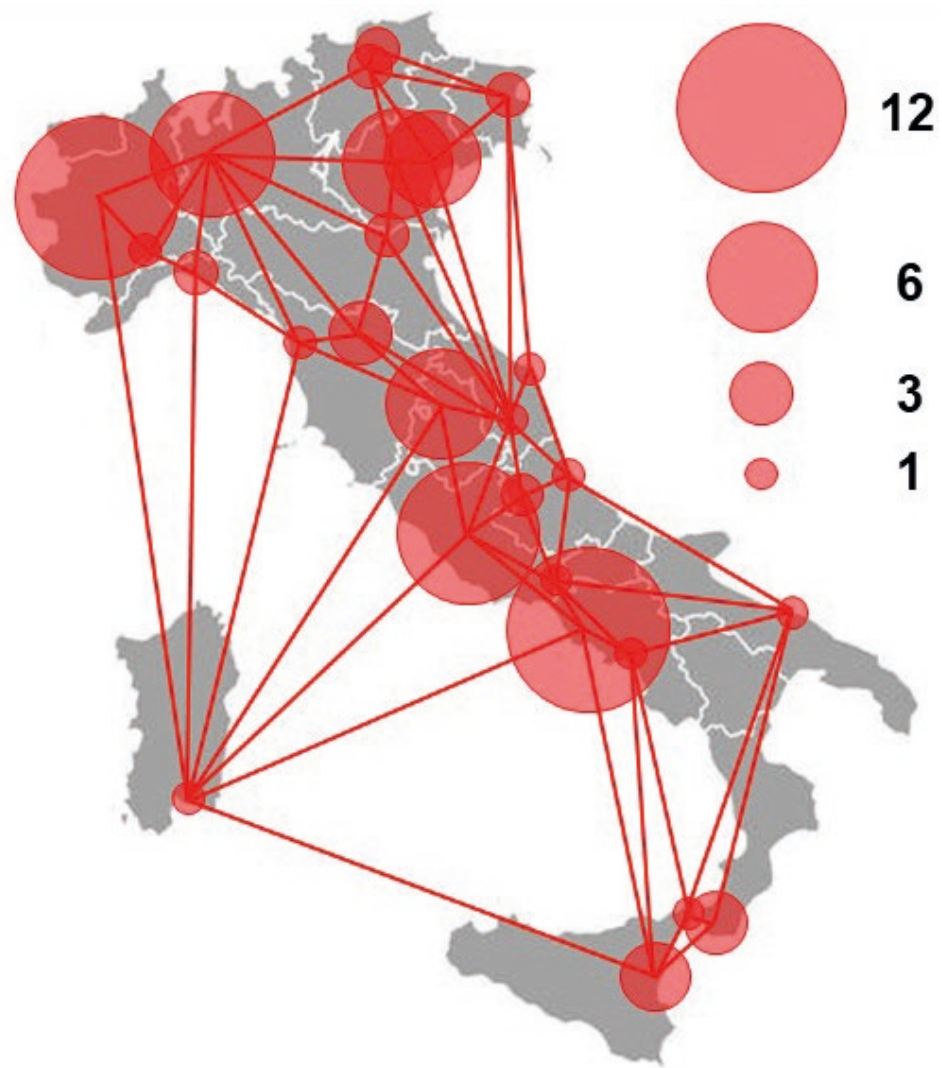

The volume that we are printing today follows the steps of the Symposium: the discussion that emerged from it has fed the proposal of the published essays, which have been subjected to peer review. In addition, the request to prepare a research paper after the meeting gave the authors the possibility to frame their research experience in an aware context, suggesting cross-references with other colleagues. The themes developed were collected in eight lines described below, which may guide future research developments.

\section{AR\&AI Theoretical Concepts}

While augmented reality has been part of the disciplinary interests of Representation for at least twenty years, the much more recent developments in artificial intelligence and the possibilities of connecting the potential of the two technologies triggered significant theoretical reflections. The contributions in this section of the book range from the historical and cultural background, to the possible challenges of Representation, opening to other areas of knowledge.

An historical excursus has been traced by Alberto Sdegno who brings to attention the concept of 'Drawing Automata' that he discusses in the sense of automata that draw. The author crosses some singular experiences like those with 'mechanical puppets', up to developments in graphic technology for digital drawing, since the first virtual reality systems and the use of artificial intelligence aimed at shape recognition. Finally, he affirms that, in his opinion, the most interesting experiments will be the ones carried out in the field of shape grammars, and in particular those in which the role of architecture is involved.

The link with disciplinary roots is explored by Francesco Bergamo who tries to answer one of the core issues of the discussion: the role of Drawing in representing and designing new epistemological models during the current Al spread, especially in relation to today's available advanced tools. He observes that Drawing always played a major role in modeling knowledge and explains that the technical achievements, deployed while facing the current challenges, should be connected with the historical and critical background pro- 
vided by the genealogies of perspective and maps. On the same line, Marco Vitali, Giulia Bertola, Fabrizio Natta \& Francesca Ronco note that the links between Representation and artificial intelligence involve many fields of architectural research, recording continuous and significant advances that require a constant update of the state of the art and a careful consideration of the role of Representation in interdisciplinary researches. For this reason, they investigate the most cutting-edge case studies in the fields of Cultural Heritage, Museums, and Digital fabrication.

Then, Giorgio Buratti, Sara Conte \& Michela Rossi explore the new frontiers of Al applied to Cultural Heritage and identify three main lines of research in this field that exploit the synergy between machine learning, big data and Al. They deal with: the development of innovative methodologies and systems to monitor and prevent damage to the cultural heritage; the experiments that focus on automatic data acquisition, such as the application of mobile technologies for three-dimensional surveying and acquisition of $\mathrm{CH}$ images; the management of large amounts of analog or digital data and information. Also Valerio Palma notices the unprecedented opportunities of advances in information and communication technologies for the documentation and analysis of architectural and urban assets. Nevertheless, he warns of the risk that the schematic understanding of reality produced by eidomatic tools, i.e. automatic recognition and classification become a black box, that is, algorithms we accept the results without questioning how these are produced or whether they can be adapted to changing needs. Then, Palma summarizes three connected research experiences he has been part of. They produced apps and prototypes exploiting geospatial databases, Al for image recognition, and AR based on model tracking, applied to architectural heritage, contributing an insight on the interpretation of the built environment form as a means to produce shared knowledge and operational outcomes.

Other authors develop broader reasoning regarding the design and ethical impact of new technologies. It is the case of Claudio Marchese \& Antonino Nastasi that observe, in the digital age, the persistence of time and space as coordinates for human presence and their intrinsic relationship with Architecture. They analyze as sort of 'scenarios' concerning prefiguration, measurements, and relationships, dealing with AI and AR combinations in a projectual perspective. Moreover, Marco Ferrari \& Lodovica Valetti analyze the ethical issues triggered by the integration of new technologies in the management of Cultural Heritage, in particular historical sites and museums, related to ontological, economic, and social aspects, trying to outline some principles able to limit these critical implications. They focus on the authenticity of reproduction, the costs of $V R$ and $A R$ systems, and individualism due to the use of personal devices. The scholars conclude that the relationship between man and machine and the concepts of real and virtual must be kept at the center of innovation and application in this field.

\section{AR\&AI Virtual Reconstruction}

Digital convergence in the last decades has favored the development of virtual reconstructions for heritage enhancement. These are connected with documentary analysis and digital survey, dialoguing both with textual, iconographic and archival sources, and with artistic, archaeological, architectural and urban consistencies. Artificial intelligence and augmented reality are intertwined with knowledge, interpretation and communication, enhancing the value of virtual reconstructions from the point of view of scientific validation, knowledge construction, and inclusion.

The dialogue with architectural literature is interwoven by Alessio Bortot, who develops his reasoning connecting the treatise of stereotomy by Monduit with AR technology with the aim to better describe the tracing of stone cutting. Starting from the interest for de La Rue stereoscopic approach, which gives the opportunity to go beyond the two-dimensional limits of paper surfaces, such as the use of pop-ups, the scholar proposes the use of AR for creating digital pop-ups. The reconstructive 3D models of the treatise plates were provided with different semantic values and, through AR, could be visualized and split into blocks, while the relationship between form and structure was shown through the use 
of a false color Fem analysis. Similarly, Cristina Càndito, Andrea Quartara \& Alessandro Meloni study the scientific sources to deal with the representation of six Keplerian stellated dodecahedrons in the decoration of an aulic room in an historical palace in Genoa. Through the virtual reconstruction of the illusory space frescoed on the vault, they illustrate in a communicative, but not simplified way, through an immersive AR experience, the connections between the Genoese decoration and the wider seventeenth-century scientific milieu.

The analysis of archival and bibliographical sources, coupled with digital survey, supports the considerations of Maria Grazia Cianci, Daniele Calisi, Sara Colaceci \& Francesca Paola Mondelli in light of the assignment by the municipality of Rome for the realization of three competition dossiers. They reflect on how and to what extent the Public Administration can now benefit from new technologies and digital tools, and conclude that although the current huge step is towards the digitization of the architectural heritage, it is also desirable to proceed towards Virtual Reality to ensure immersive design for competitors. Also Maurizio Marco Bocconcino \& Mariapaola Vozzola present remarks that focus on analysis at urban scale, in order to propose, in this case, solutions aimed at safeguarding memory. They show the methodological framework for a research project on conservation and care of the memory of changing urban contexts and highlight the need of implementation of integrated digital archives with interactive and hypertextual navigation between the assets themselves.

Virtual reconstructions, linked to AR and Al technologies, are particularly developed in the archaeological field. It is the case of the research by Riccardo Florio, Raffaele Catuogno, Teresa Della Corte \& Veronica Marino. They set up a methodology to deepen the investigation on the current consistencies of the Roman bath of Cumae that, through the preparation of a suitable neural network managed by Al processes, are able to formulate reconstructive hypotheses with scientific authority. Moreover, through the adoption of web-based and open source technologies and the applications of AR and interactive digital visualization, multiple information are associated with the 3D model. Also Maurizio Perticarini \& Chiara Callegaro deal with archaeological heritage and propose the creation of an adaptive AR app with the aim to enhance through cultural tourism the vestiges of ancient buildings hidden by urban building development of Padua. The workflow identifies a path through the city and connects the analyzed and represented artifacts through documents, digital survey, BIM modeling and, finally, AR app, foreseeing further insights through artificial intelligence to differentiate the contents according to the user's preferences.

Finally, Antonio Calandriello exploits ICTs, i.e. digital reconstruction, virtual and augmented reality, video production, and application development, aimed at the enhancement of a Renaissance palace in Padua. In this way, it is possible to increase the inclusiveness and raise the level of use, giving visitors the opportunity to virtually visit the building and its precious frescoed halls, which the public cannot visit.

\section{AR\&AI Heritage Routes}

Built and environmental heritage are very often made up of a series of artefacts and elements that take on a particular value in their constitution as a system. The construction and use of heritage routes can profitably avail of augmented reality and artificial intelligence technologies for the construction of knowledge and its sharing as well as for flow control and route recommendation. The researches presented in this section demonstrate the desire to create closer links between the real and the virtual sets, realizing a real continuum between them.

In this sense moves the work of Stefano Brusaporci, Pamela Maiezza, Alessandra Tata, Fabio Graziosi \& Fabio Franchi dealing with the idea that a smart heritage could be realized through VR/AR/MR combined with Al applications. Their observations are based on a wide experience on the territory of L'Aquila that highlights the potential of the so-called 'phygital' with the protection and enhancement of assets often characterized by important elements 
of fragility. In the same direction, although referring to environmental heritage, Alessandra Pagliano, Greta Attademo \& Anna Lisa Pecora propose a systematization of the knowledge of the Phlegraean Fields Park, through surveys and 3D models, integrated by the use of different digital technologies, AR in particular. Each site becomes the node of a network of thematic routes, traced starting from the major attractions of the area. The goal is to define a hybrid landscape, made of site specific visits and digital experiences, that generate a new model of inclusive museum, configuring cultural relationships between physically distant places. Also Andrea Rolando, Domenico D'Uva \& Alessandro Scandiffio work on environmental heritage enhancement. They aim at investigating the potential of image segmentation technology, based on web application, for measuring the spatial quality of slow routes. The method has been applied to some stretches of slow-mobility routes that are localized along a fragile coastal landscape of southern Italy. Such an update of the tool could take advantage of Machine Learning systems, namely the ability to learn through analysis, to improve its precision and reliability.

The research of Gerardo Maria Cennamo is engaged in the so called 'open air museums' linking in a single path a series of cultural stops. The author refers to two ongoing experiences in Naples and Rome where, thanks to GPS systems it is possible to develop dynamic paths of perception of the archaeological and architectural heritage, integrated by augmented reality applications that realize an active involvement of the visitor through pedestrian and vehicular itineraries.

Educational aims address the cutting-edge research by Alessandro Luigini, Stefano Brusaporci, Alessandro Basso \& Pamela Maiezza that present a project addressed to the documentation, investigation of architectural values, and valorization of a Sanctuary in Pescara, through an application of augmented reality technology, able to visualize the previous configuration of the church, enhanced by an artificial intelligence-based tracking application. The awareness-raising program includes online communication and activities with local schools. Also Serena Fumero \& Benedetta Frezzotti describe a didactic experience related to the use of AR in the promotion of heritage sites. The low cost and the relative ease of creating content of the cultural experience will encourage a similar approach in many other heritage sites.

The heritage route set up by Marinella Arena \& Gianluca Lax develops a strategy to guide users and scholars in the Byzantine iconographic world, highlighting the elements that contribute to recognizing the sacred figures represented. The artificial-intelligence-based technique used to analyze 19 images of Saint Nicholas set up some preliminary strategies for the morphological classification of the iconography through the identification of the proportional relationships between the parts of the face.

In an original way, Ornella Zerlenga, Vincenzo Cirillo, Massimiliano Masullo, Aniello Pascale \& Luigi Maffei create a philological heritage route investigating the ephemeral architecture of the eighteenth century in Naples. The goal is an unprecedented representation, modeling and fruition project, accompanied by a graphic visualization of the places to better understand the architectural and urban space. An Augmented-Reality application prototype makes it possible to disseminate the compositional elements of the celebrations to a non-specialist public.

Finally, Giorgio Verdiani, Ylenia Ricci, Andrea Pasquali \& Stéphane Giraudeau set up a real route between different research experiences personally carried out, that links together digital modelling of no longer and still existing architectures with $A R, V R$ and $X R$ experiences. Not only in the will of capturing the users, but in the true belief that Digital Heritage is an occasion allowing an easier reading and an option for spreading the knowledge of Cultural Heritage.

\section{AR\&AI Classification and 3D Analysis}

$A R$ \& Al classification and 3D analysis is an increasingly important area of research through data segmentation and attribution of specific connotations, supporting the architectural analysis process. In particular, the research focus shifts from 3D acquisition to data processing, defining hierarchical-oriented information systems. This strategy implements the geometrical data structures for reality-based and interpretative definition of model, opti- 
mizing the model communication passage through AR/MR/VR systems. The papers cover the entire process, from the treatment of the survey data to the Scan-to-BIM passage, up to the building's analysis and digital heritage enhancement.

The first group of articles is oriented towards photogrammetric techniques underlying data processing. Marco Limongiello \& Lucas Matias Gujski work directly on data acquired by UAV imagery. They suggest an innovative approach that can support survey methods by applying Al algorithms, improving the accuracy of extracted point clouds. Some parameters are analyzed through a Self-Organizing Map (SOM) to reach a compromise between the variable values, the noise reduction, and the 3D model definition, allowing a fast data clustering. Similarly, Andrea Tomalini, Edoardo Pristeri \& Letizia Bergamasco start from the UAV imaging approach and suggest the use of a photogrammetric model to generate new image datasets for training Machine Learning (ML) algorithms and recognize built heritage. They apply Physically Based Rendering (PBR) tools to extend the image datasets with artificial data. In the end, Pierpaolo D'Agostino \& Federico Minelli propose a methodology for the automated delineation of brick masonries from images to a vector representation. The edge detection and vector delineation of brick joints allow a brick clustering for masonry classification, implemented in AR visualization.

The second group is oriented to the segmentation of point clouds acquired by 3D scanners for data classification with the aim to study architecture. Michele Russo, Eleonora Grilli, Fabio Remondino, Simone Teruggi \& Francesco Fassi present an iterative multi-level and multiresolution classification process that improves the learning activity based on a supervised automatic Machine Learning approach to optimize 3D point cloud classification by a hierarchical concept. This strategy requires few annotated 3D data while very detailed semantic segmentation results can be achieved. Paolo Clini, Roberto Pierdicca, Ramona Quattrini, Emanuele Frontoni \& Romina Nespeca suggest an innovative approach based on HBIM existing models to improve the segmentation of 3D point clouds through the Deep Learning approach. The proposed methodology facilitates Scan-to-HBIM passage, generating a sufficiently large data set of synthetic clouds to train the neural network. The last paper referred to this cluster is a work by Federica Maietti, Marco Medici \& Ernesto ladanza and focuses on Al and 3D point cloud models, showing a new integration within the BIM environment, image classification algorithms, point cloud segmentation, and representation models. This paper presents a preliminary experience focused on the Al for processing the large amount of data obtained from digitization processes applied to the Architectural Heritage.

In the last three research papers, the modeling activity based on acquired geometrical data assumes a strategic value. In such a sense, Elisabetta Caterina Giovannini presents an extended analysis of the current methodologies and workflows for data modeling in Architecture, deepening the use of standards, descriptive metadata, and ontologies for Cultural Heritage representation. Furthermore, this research underlines the complexity of creating conceptual models able to manage three-dimensional data and descriptive metadata on architectural heritage documentation.

The last two articles share common themes of museums and IT, though explored from two different points of view. Massimiliano Campi, Valeria Cera, Francesco Cutugno, Antonella di Luggo, Domenico lovane \& Antonio Origlia propose a methodology of collecting, analyzing, and modeling multimodal data, helpful in designing virtual agents in 3D museum environments. This research is part of the national CHROME project, which deepens the interconnections between representation, survey, and information technology domains. The architecture focuses on graph databases and 3D models, using modern peripheral devices and third-party services for capturing and analyzing input signals; it also integrates probabilistic decision-making systems for controlling interaction in 3D environments. Besides, Marco Giorgio Bevilacqua, Anthony Fedeli, Federico Capriuoli, Antonella Gioli, Cosimo Monteleone \& Andrea Piemonte focus the attention mainly on the communication museum strategy. They suggest a new visit path based on immersive experiences of video mapping, VR/AR, sound immersion, informative totems, audio-visual supports, and multisensory activities. The project aims to investigate the potential in applying new AR/VR technologies to enhance virtual tours and interpret 3D models of real environments. 


\section{AR\&AI Urban Enhancement}

This section is dedicated to research that refers mainly to the urban space and the territorial scale, considering also building knowledge connected to the territory and the architecture culture. The central topic of this section is the cultural and sensory itinerary, allowing experiences that go beyond the visible. The use of immersive and augmented virtual reality techniques creates a link between the tangible and intangible, memory and existing real space, underlying the construction logic, the building itineraries spreading knowledge. Both urban systems and single buildings are spatial and historical landmarks. Their disappearance or damage may erase the relation between present and past, causing a sense of disorientation. The first articles explore ARVR methodologies for the memory recovery of places destroyed by earthquakes. Giuseppe Amoruso, Polina Mironenko \& Valentina Demarchi propose a path of memory re-appropriation through multimedia installations, emphasizing the evocative interaction between reality, memory, and reproduction of the intangible to promote collective memory. Thus, the real environment becomes a temporary and experiential museum, expanding the immaterial dimension of accessibility discovery. Besides, Caterina Palestini \& Alessandro Basso suggest a solution to promote the use of the historical buildings, activating at the same time the dynamics of cultural regeneration in the area, using AR+Al systems for a tour that can show the urban architectural evolution, monitoring and enhancing the knowledge and the transformations undergone by the architectural heritage.

Concerning urban routes, the experiential factor plays a central role. The experience occurs mainly through the human vision, which is dominant in AR/MRVR development without excluding other senses. Alessandra Pagliano presents a project aiming at preserving the memory of murals using AR. In this way, the narrative message of the old murals is expanded thanks to the digital multimedia contents, reinforcing the storytelling, the cultural connection, and the memory of the place. On the other hand, Fabio Bianconi, Marco Filippucci \& Marco Seccaroni suggest using EEG helmets synchronized to GPS to collect human cognitive conditions and transform them into representations that reflect the people's feelings during their itinerary. This research aims to define methodologies based on interdisciplinary interpretative criteria to understand the human-environment impact, directing design choices to improve wellbeing. As mentioned earlier, large architectural spaces can be compared with those at urban scale, especially if the aim is to communicate and promote them through a more profound knowledge. This is the case of Marco Canciani, Giovanna Spadafora, Mauro Saccone \& Antonio Camassa, who present two AR applications about important architectonic space, with the final aim to develop some AR multimedia content linked to semantic concepts. The proposed experiences use AR to share the analyses carried out on drawings and canvas, unveiling the constructive meaning of space.

The last cluster is dedicated to communication and promotion of local assets and retails, always framed through knowledge itineraries. Paolo Belardi, Valeria Menchetelli, Giovanna Ramaccini, Margherita Maria Ristori \& Camilla Sorignani present the concept of "Augmented Retail," representing a strategy to achieve an Augmented Identity, changing the retail space from a 'product window' to an 'experiential theatre'. This research aims to open new perspectives based on AR and Al to enhance local identity promoting itineraries of historic shops. Regarding local assets, Daniele Rossi \& Federico O. Oppedisano, illustrates the potential of AR in the valorization of food and wine heritage. They highlight the playful interactive capacities of this technology shifting towards renewed communication models that aim to extend the cultural offer in an increasingly rapid and immediate form.

\section{AR\&AI Museum Heritage}

In the digital era, museums have seen a significant expansion of spaces and tools for the dissemination of knowledge. In this sense, the digital takes on multiple roles precisely articulating itself between Al and AR for new ways of disseminating and communicating Cultural Heritage. In this sense, Massimo Barilla \& Daniele Colistra are involved in the creation of an immersive and interactive room - Nello Scill'e Cariddi - in which the marine environment of the 
Strait of Messina is used as the first experimental scenarios to develop and test technology Al/AR. The room, therefore, transforms film collections, integrating them with the production of specific images, into virtual environments containing structured catalogs and into interactive installations for educational, playful, scientific and popular use. Francesco Borella, Isabella Friso, Ludovica Galeazzo, Cosimo Monteleone \& Elena Svalduz then deal with the cultural interfaces that make a video installation which is also immersive and interactive. Their aim is to keep a high and active visitor's attention, in the video dedicated to the Academia insula of Venice several short-term sections have been introduced, which can be interrogated and activated by sensors, while a Sound Shower system facilitates the understanding of the multimedia story by listening to the voice of a storyteller.The contribution of Laura Carlevaris, Marco Fasolo \& Flavia Camagni is also fundamental, dealing with the options provided by the recent applications of Augmented Reality (AR) to the knowledge and enhancement of a cultural asset: the perspective in wood inlays. The intention is therefore to use perspective in $A R$ ambient as the ideal tool to virtually experience the space represented in the exhibited decoration. The creation of an experiential path linked to perspective, organized by a sensitive narrative that begins in the very act of its use, to allow the observer a critical interaction dependent on the process of knowledge of the works and their evoked spaces. This is what Gabriella Liva \& Massimiliano Ciammaichella propose for the perspective analysis of some canvases by Jacopo Robusti. Furthermore, for the enhancement of Cultural Heritage it is important to explore the potential of the combined use of Augmented Reality (AR) and rapid prototyping. Giuseppe D'Acunto explores how these innovative methodologies can be effective in the creation of museum installations able not only to show the contents of an exhibition in an original and attractive way, but also to recover the memory of a place by reconstructing the original position of the environment, as in the case of the installation of Palazzo Grimani, where the sculptures have moved from their original position over the centuries. These aspects linked to the relationship between AR and VR for the installations are addressed by Giuseppe Di Gregorio, who highlights the workflow produced for the realization of the $3 \mathrm{D}$ models of one of the three churches in the rocks of the necropolis of Pantalica, an Unesco site. In this sense, the ongoing process to achieve the realization of VR and AR models to use with three different levels of immersive reality is important: normal commercial viewers, different types of Oculus viewers, or in special virtual rooms Cave Automatic Virtual Environment (CAVE). In this sense, the paper of Elena Ippoliti also deals with the research experiences of the last I 5 years in which the potential of digital technology, and of Augmented Reality in particular, has been tested to enhance Cultural Heritage. This research outlines the value of preliminary digital simulation for form-finding, both in terms of the actual final configuration of the project and in terms of the less tangible aspects of the efficiency of building. As a result, representation takes on a new position as the 'place' of the model. The dynamic passage between real and virtual in a spatial model helps in representing intangibly, with high reliability, what is concretely abstract. The challenge of the use of Cultural Heritage by subjects with sensory limits has also become fundamental for museum installations. Franco Prampolini, Dina Porpiglia \& Antonio Gambino propose the 3D survey of all the main finds to be exhibited with analytical photo-modeling techniques, their scientific cataloging, the creation of a website with a high interactivity content and an application that allows sharing extended information with blind people, through the combined use of analog 3D models and AR creation software. The topic of facial recognition technologies - already used today in many applications, to support security systems in sensitive buildings - is another developing theme for museums or art galleries. Indeed, Paola Puma \& Giuseppe Nicastro describe an experimentation in a museum of a research in order to provide a tool capable of interpreting the reaction of a user in front of a work of art, proposing a responsive information content with what is manifested, through facial expressions. Finally, it is important to highlight how generative design is now fundamental for the creation of interactive digital configurations, aimed at the creation of artistic languages and for the representation of dynamic contexts. In this sense, Leopoldo Repola, Nicola Scotto di Carlo, Andrea Maioli \& Matteo Martignoni have integrated a narrative process, linking aspects of recording the movement of bodies in water with the principles of data visualization. 


\section{AR\&AI Building Information Modeling and Monitoring}

$A R$ \& $\mathrm{Al}$ are currently playing an important role in the advanced implementation of BIM with special reference also to the monitoring of architectural and engineering artifacts. And there are many applications also in terms of Scan-to-BIM, such as the essay of Vincenzo Bagnolo, Raffaele Argiolas \& Nicola Paba which present the first results of an ongoing research on the advantages of implementing computational modeling in Scan-to-BIM processes for the representation of historical architecture in AR and VR applications for educational and communication purposes. But if we consider that the conservation and regeneration of the existing built heritage is still characterized by an inefficient management of time and costs, throughout the life cycle, it is central the research proposed by Marcello Balzani, Fabiana Raco \& Manlio Montuori which focus on the development of integrated digital solutions for the acquisition, modeling and visualization of data relating to the building and construction supply chain. At the same time Fabrizio Banfi highlights the convergence of innovative methods, latest generation of technologies and software applications for the representation, archiving, transmission of the material and immaterial values of architecture; for these reasons, he researches the development of a cloud-based open source BIM platform and XR/AI projects able of sharing a knowledge process based on new levels of interactivity and digital creativity. Carlo Biagini, Ylenia Ricci \& Irene Villoresi also highlight how, in recent years, the application of BIM to Cultural Heritage has led to the development of solid operating methods that have enabled more efficient information management. They then tested whether BIM models can be exploited to create immersive experiences in digitally simulated environments, setting new ways of viewing and evaluating the built space. In this sense it is important to test the accuracy of the solutions adopted using BIM models, in a representation of the built structure that is also able to summarize the qualities to be detected. Fabio Bianconi, Marco Filippucci \& Giulia Pelliccia, with their research, have developed some case studies in the field of wooden constructions, inserting themselves in a framework that emphasizes the relationship between simulation and realization. The introduction of automation methodologies through the use of deep learning of BIM modeling starting from different types of formats, such as digital processing of paper documents and CAD formats, is also important for the relationship between BIM and Al. In this sense, David Campagnolo \& Paolo Borin emphasize as a proof of concept of a possible contribution that a technique currently barely adopted in the architectural field - such as deep learning - can lead to the design, in particular in the creation of the information model, an activity that today takes time. If we then consider that in the digital age the construction industry has seen significant changes in the design, construction and learning of spatial processes through new technological systems, it is important to highlight how these processes influence the management and the way in which data are collected, cataloged and monitored using sensors and connected users. Matteo Del Giudice, Daniela De Luca \& Anna Osello therefore define new safe and resilient digital models combined with interoperable methods that minimize the impacts of our built heritage during its life cycle. In this direction we find the essay of Raissa Garozzo who proposes a new methodological approach for the evaluation of the health status of railway bridges in masonry based on the definition of image-based and Al-driven investigation protocols useful for the creation of semiautomatic H-BIM models. Similarly, the research of Federico Mario La Russa which provides an overview of the evolution of the VPL and an application case concerning the classification of seismic vulnerability indices with Al. An important topic involved in this volume regards also energy monitoring that can be implemented in an advanced way with BIM as in the research of Marco Filippucci, Fabio Bianconi \& Michela Meschini who analyze, in particular, those buildings that, even if of different eras and construction technologies, require energy requalification. Finally, the contribution of Assunta Pelliccio \& Marco Saccucci proposes a methodology (D.V.M.R., acronym for Design, Virtualization, Modeling, Reproduction), which in four temporally consequential phases builds a tool able of providing territorial, environmental, architectural and historical information of a particular case study (an industrial building) but extendable to the built heritage. 


\section{AR\&AI Education and Shape Representation}

A particular area of development of AR \& Al could be certainly linked to educational purposes. Indeed, there are many repercussions that we can apply to students' training especially for aspects concerning inclusion and support. For example, the difficulties involving clinical autistics are mainly related to the perception deficit, therefore VR/AR \& Al can become a valid support for people with ASD, improving relationships with space and people. Anna Lisa Pecora, in this sense, tries to provide a guiding tool for a human-centered VR design. So, at an academic level of education, AR \& Al may facilitate the preparation in faculties such as Architecture and Engineering. For example, Emanuela Lanzara \& Mara Capone propose how to improve a dataset of generative algorithmic definitions capable of returning an optimized "semi-ideal" curve that best fits a generic profile based on reality, starting from some of its points, with interesting effects in the field of education. Therefore, learning through the direct experimentation of "intelligent" models - in their variety of manifestations and hybridizations - is undoubtedly a very powerful aid in the acquisition of knowledge. This is what Marta Salvatore, Leonardo Baglioni, Graziano Mario Valenti \& Alessandro Martinelli assert, entering into the specific role of the architectural configuration: testing or experimenting with models helps not only to understand the shape of existing architecture, but even more to imagine and design new buildings. In this sense, the essay proposed by Roberta Spallone \& Valerio Palma presents methodologies, objectives and some of the results of a university course - Techniques of Digital Representation - in which the interaction between the different digital representation techniques in relation to AR and Al technologies is developed, providing tools for investigating critical aspects. Finally, it is important to underline the repercussions that training in these terms has for architecture, engineering and construction (AEC) industries: Alberto Tono, Meher Shashwat Nigam, Stasya Fedorova, Amirhossein Ahmadnia \& Cecilia Bolognesi test algorithms for 3D reconstruction from a single image, specifically for building envelopes, with end-to-end geometric deep learning approaches. At the same time, the importance of this kind of apprenticeship can be recognized in the touristic industry, from a communication point of view, as Maria Linda Falcidieno, Maria Elisabetta Ruggiero \& Ruggiero Torti affirm: their research introduces some insights and proposals shared with the operators of the sector and aimed at the intelligent reformulation of approaches and languages in order to bring potential customers closer to the Cruise Experience.

\section{Notes}

[I] Francesca Fatta is the actual president of UID (Unione Italiana del Disegno), an association that gathers all the Italian scholars of Representation.

\footnotetext{
Authors

Andrea Giordano, Dept. of Civil, Environmental and Architectural Engineering, University of Padua, andrea.giordano@unipd.it Michele Russo, Dept. of History, Representation and Restoration of Architecture, Sapienza University of Rome, m.russo@uniromal.it Roberta Spallone, Dept. of Architecture and Design, Politecnico di Torino, roberta.spallone@polito.it
} 
